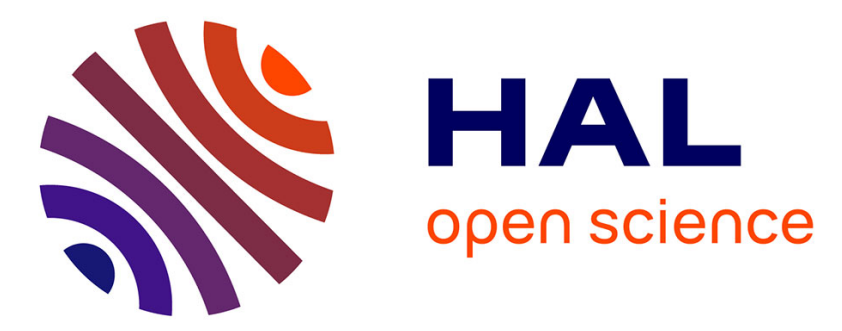

\title{
Nationalité et citoyenneté en Allemagne aujourd'hui Riva Kastoryano
}

\section{To cite this version:}

Riva Kastoryano. Nationalité et citoyenneté en Allemagne aujourd'hui. Vingtième siècle. Revue d'histoire, 2001, 70, pp.3-17. 10.3917/ving.070.0003 . hal-01016936

\section{HAL Id: hal-01016936 \\ https://hal-sciencespo.archives-ouvertes.fr/hal-01016936}

Submitted on 1 Jul 2014

HAL is a multi-disciplinary open access archive for the deposit and dissemination of scientific research documents, whether they are published or not. The documents may come from teaching and research institutions in France or abroad, or from public or private research centers.
L'archive ouverte pluridisciplinaire HAL, est destinée au dépôt et à la diffusion de documents scientifiques de niveau recherche, publiés ou non, émanant des établissements d'enseignement et de recherche français ou étrangers, des laboratoires publics ou privés. 


\title{
Nationalité et citoyenneté en Allemagne aujourd'hui
}

In: Vingtième Siècle. Revue d'histoire. N70, avril-juin 2001. pp. 3-17.

\begin{abstract}
Nationality and Citizenship in Germany Today, Riva Kastoryano.

In 1999, Germany broke with its conception of nationality as the right based on place of birth, recognized since 1913, and even with its conception of the nation going back to Herder and Fichte. This event, as significant for it as for Europe, is put into historicalperspective. It shows Germany as a country of immigration, a multicultural society in which participation could lead to citizenship, a nation and a state in which new citizens upset legacies and representations and in which double nationality is not a panacea. The strength of immigration, Turkish above all, bas forced the country to negotiate its own identity differently and to seek a democracy beyond the one set up in 1949.
\end{abstract}

\section{Résumé}

Nationalité et citoyenneté en Allemagne aujourd'hui, Riva Kastoryano.

L'Allemagne vient de rompre en 1999 avec le droit du sang qu'elle reconnaissait depuis 1913 et même avec sa conception de la nation qui remontait à Herder et Fichte. Cet événement considérable, pour elle comme pour l'Europe, est mis ici en perspective historique. Ce rappel dévoile une Allemagne pays d'immigration, une société multiculturelle où la participation tente de déboucher sur une citoyenneté, une nation et un État où les nouveaux citoyens bousculent les héritages et les représentations et où la double nationalité n'est pas une panacée. C'est bien la force de l'immigration, et d'abord turque, qui tout au long a contraint ce pays à négocier différemment sa propre identité et à rechercher un au-delà de la démocratie constitué en 1949.

Citer ce document / Cite this document :

Kastoryano Riva. Nationalité et citoyenneté en Allemagne aujourd'hui. In: Vingtième Siècle. Revue d'histoire. $\mathrm{N}^{\circ} 70$, avril-juin 2001. pp. 3-17.

doi : $10.3406 / x x s .2001 .1341$

http://www.persee.fr/web/revues/home/prescript/article/xxs_0294-1759_2001_num_70_1_1341 


\title{
NATIONALITÉ ET CITOYENNETÉ EN ALLEMAGNE AUJOURD'HUI
}

\author{
Riva Kastoryano
}

L'Allemagne vient de rompre en 1999 avec le droit du sang et même avec une certaine conception de la nation qui remontait à Herder et Fichte. Cet événement considérable, pour elle comme pour l'Europe, est mis ici en perspective historique. Ce rappel dévoile une Allemagne pays d'immigration, une société multiculturelle où la participation tente de déboucher sur une citoyenneté, une nation et un État où les nouveaux citoyens bousculent les héritages et les représentations pour aboutir à une nouvelle définition de l'unité.

D epuis le $1^{\text {er }}$ janvier 2000 , tout enfant né sur le territoire allemand de parents étrangers eux-mêmes nés sur le sol allemand, ou arrivés à l'âge de 8 ans, est allemand à sa naissance. Cette nouvelle loi marque ainsi un tournant historique en remplaçant le droit du sang et de l'affiliation, en vigueur depuis 1913, par le droit du sol. Son vote au Bundesrat en mai 1999 est le résultat de longues négociations entre les partis politiques, d'un côté, et l'État fédéral et les immigrés, de l'autre, les partis politiques s'accordant tous sur la nécessité d'intégrer les étrangers installés sur le territoire allemand dans la communauté nationale, les immigrés, quant à eux, revendiquant le droit de maintenir leur nationalité d'origine comme condition de passage à la nationalité allemande. Comment, dans ce contexte, combiner idéologie et réalité, rhétorique et droit qui ensemble renvoient à un compromis entre morale politique et limites juridiques ?

La question de la citoyenneté s'est imposée à l'Allemagne avec l'arrivée massive des Aussiedler après la chute du mur de Berlin et l'écroulement du monde soviétique. Venus d'Europe centrale et de Russie, les Aussiedler ont été naturalisés de fait et de jure sur preuve de leur affiliation et de leur appartenance au "peuple "allemand, alors que des populations étrangères installées en Allemagne depuis plus de trente ans, socialisées dans les institutions locales et nationales restaient maintenues à l'écart de la communauté politique, à cause précisément de leur ancêtre, et en vertu du principe fondamental du droit du sang, jus sanguinis. La naturalisation discrétionnaire des étrangers pose, par conséquent, une question de morale politique qui a accéléré le débat sur le droit à la citoyenneté des "étrangers de sang ". Ce débat s'est amplifié autour de la double nationalité, rejetée par la Constitution et revendiquée par les Turcs comme condition d'accès à la citoyenneté égale et a entretenu des émotions de la part de l'opposition et de l'opinion publique, conduisant Schröder, qui l'avait incluse dans le projet de loi, à la retirer.

L'idée de la double nationalité introduit de fait une distinction entre citoyenneté et nationalité, deux concepts interdépendants et " interchangeables " ${ }^{1}$ dans le cadre de l'État-nation qui se définissent comme l'appartenance de l'individu à une communauté politique. Cette appartenance prend forme à travers les droits (sociaux, politiques, culturels) et les devoirs qu'incarne

1. J. Leca, - Nationalité et citoyenneté dans l'Europe des immigrations :, dans J. Costa-Lascoux, P. Weil. Logiques d'Etat et immigration en Europe, Paris, Kimé, 1992. 
l'idée même de la citoyenneté. Mais l'appartenance ne se limite pas au droit. L'acte juridique qui concrétise ce principe implique l'inclusion de "l'étranger " dans la communauté nationale avec laquelle il est censé partager les mêmes valeurs morales et politiques. Plus encore il est censé adopter, voire s'approprier, les références historiques comme preuve d'adhésion complète et de loyauté aux principes fondateurs de la nation, qui d'après Weber est la seule communauté née de la modernité.

Telles sont du moins les attentes. Elles s'appuient sur les principes fondateurs de la formation de l'Etat-nation, de ses traditions politiques, bref de ce qui est devenu son identité nationale. Cela aussi bien en Allemagne qu'en France, dans deux républiques marquées par des histoires différentes et représentées comme deux " modèles " de citoyenneté et de nationalité systématiquement opposés : la France serait l'exemple même de l'État-nation et se conçoit comme universaliste tant elle est assimilationiste et égalitaire, et l'Allemagne, une nation exclusiviste qui privilégie les ancêtres communs et l'appartenance à une même communauté culturelle. Ces différences servent de fond rhétorique, alimentent les débats publics et touchent de façon évidente aux structures mentales elles-mêmes. Elles agissent en même temps sur les pratiques institutionnelles et déterminent les principes juridiques d'accès à la citoyenneté : la France privilégie la socialisation sur le territoire français et dans les institutions, et l'Allemagne maintient un droit qui demeure exclusivement rattaché au culte des " ancêtres " .

Mais les réalités sociales rapprochent les deux pays dans les discours et dans les lois ${ }^{2}$. La citoyenneté allemande ne peut être détachée de sa conception politique et inversement la citoyenneté française ne

1. Cf. W.R. Brubaker, Citizenship and Nationbood in France and Germany, Cambridge, MA Harvard University Press, 1992 ; L. Dumont, L'idéologie allemande. France-Allemagne et retour, Paris, Gallimard, 1991. peut faire abstraction de son élément culturel transmissible. Face aux modes d'organisation communautaire des populations immigrées des années 1960 et leurs revendications de reconnaissance d'une ou des spécificités culturelles, les deux États expriment de la même façon un sentiment de " suspicion "vis-à-vis de l'immigrant, un sentiment qui surgit lors de tous les débats sur l'immigration et sur la citoyenneté et qui traduit incontestablement l'appréhension de la classe politique et de l'opinion publique de voir la nationalité " désacralisée " par une "citoyenneté pour les papiers ", un droit dépourvu d'identité. Les arguments qui alimentent les débats publics sont puisés dans l'attachement, réel ou imaginé, des " immigrés " ou "étrangers " à leur État d'origine, vécu comme identification à une communauté nationale et religieuse à l'intérieur de la communauté politique. Alors qu'en France la rhétorique récuse toute appartenance politique à une autre communauté que la communauté nationale, en Allemagne la Constitution exige la renonciation à la nationalité d'origine lors de la naturalisation des étrangers comme preuve de leur adhésion " complète "à la nation allemande. C'est là que réside le fond du débat sur la double nationalité qui a mobilisé les passions et les émotions lors de la préparation et du vote au Parlement de la nouvelle loi en janvier 1999.

Les débats sur la citoyenneté renvoient aux droits et identités, aux valeurs morales et politiques, ainsi qu'au partage des responsabilités civiques à l'intérieur d'une même communauté politique ${ }^{3}$. Ils ouvrent pas conséquent la voie à des négociations identitaires entre États et immigrés ${ }^{4}$. Pour les États, il s'agit de négocier de nouveaux

2. Pour une convergence des lois sur la citoyenneté, cf. $\mathrm{P}$. Weil, R. Hansen, Citoyenneté et nationalité en Europe, Paris, La Découverte 1999.

3. Cf. N.M.J. Pickus, Immigration and Citizenship in the $21^{\circ}$ Century, Boston, Rowman and Littlefield $\mathrm{Pb}, 1998$ cf. p. 18

4. R. Kastoryano, La France, l'Allemagne et leurs immigrés. Négocier l'identité, Paris, Armand Colin, 1997. 
modes et de nouveaux moyens d'inclusion des populations issues de l'immigration dans la communauté politique sur la base d'un équilibre différent entre des structures communautaires qui prennent forme et les institutions nationales. À partir du principe d'égalité, il s'agit, pour les individus ou les groupes, de lutter contre toute forme d'exclusion, qu'elle soit politique, sociale ou culturelle, par lequel s'exprime l'attachement à la fois à la communauté nationale et à une identité collective autre que nationale. Cela remet nécessairement en cause le lien classique établi entre la communauté culturelle et l'appartenance politique, entre nation et État, entre nationalité et citoyenneté, les premières comme source d'identification, les deuxièmes comme droit de participation civique avec des droits égaux. C'est cette tendance qui se trouve aujourd'hui à l'origine des changements dans la conception de la citoyenneté et de rhétorique dans les États démocratiques : une citoyenneté avec des références identitaires multiples, mais avec la même responsabilité civique vis-à-vis de la communauté politique, remplaçant ainsi le "rêve de l'unité culturelle " par le "rêve d'une unité politique ".

\section{O LE RÊVE D'UNITÉ ALLEMANDE}

Le rêve d'unité nationale est fortement lié au romantisme allemand. Fichte, dans son Discours à la nation allemande, insiste sur cette idée d'unité culturelle de la nation allemande. Unité culturelle, unité de la langue (que prône Herder) ont nourri le rêve allemand d'unité nationale ; elles ont circonscrit l'identité collective à tous ceux qui partageaient les mêmes ancêtres, la même langue et la même culture. Le nationalisme allemand inspiré de Fichte a été parallèlement le produit d'une résistance à la " domination " étrangère, accompagné d'élans et de passions. Unité, droit et liberté : Einigkeit und Recht und Freibeit... telles deviennent les valeurs déterminantes de la nation. Elles apparaissent dans le "Chant pour l'Allemagne" (Deutschlandlied) du poète August Heinrich Hoffmann von Fallersleben; elles sont reprises en 1952, dans l'hymne national de la République fédérale.

Le romantisme allemand est né au début du $19^{\mathrm{e}}$ siècle en réaction à la France, aux idées révolutionnaires qui ont conduit l'Allemagne à un repli " communautaire ". En effet, le romantisme allemand, en privilégiant le sentiment d'appartenance à une culture et à un peuple, se heurte à la rationalité qui a inspiré la Révolution et ses valeurs universelles qu'il rejette. Contre le religieux rationnel qui caractérise les Lumières, et où Fichte voit la raison de la défaite de la Prusse, le romantisme réévalue l'élément religieux de la cohésion sociale. Alors qu'en même temps les réformateurs prussiens inspirés de la Révolution française lancèrent les premières ébauches de l'État de droit, les romantiques se réfèrent avec nostalgie au christianisme qui unifiait les peuples d'Europe au Moyen Âge, "la seule époque de l'histoire allemande où cette nation connut une existence brillante et glorieuse, avec le rang qui lui revient en tant que peuple souche " .

La distance avec les Lumières est exprimée par la notion de Volksgeist (esprit du peuple) et devient l'élément constitutif de la nation allemande ${ }^{2}$. Se développe ainsi l'idée de Volksnation, nation du peuple. Une telle conception de la nation nourrit la crainte d'une perte d'identité et exprime un refuge dans le temps tant elle reste enracinée dans le passé. Le passé, c'est le Saint Empire Romain, les ancêtres, le sentiment d'appartenir à une communauté d'origine. Cette communauté est "imaginée " à partir des liens organiques entre les individus partageant la même culture et s'exprime par l'appartenance au peuple allemand, même géographique-

\footnotetext{
1. Discours de Fichte, repris par A. Renaut.
}

2. R. Brubaker, Citizenship..., op. cit., p. 9. 
ment dispersé dans des royaumes n'ayant aucun réseau de communication entre eux. La référence au peuple allemand, Das deutsche Volk, met donc en évidence une appartenance avant tout ethnique. Une telle conception exclut toute différence de culture : l'unité culturelle et le caractère organique de la communauté nationale se trouvent inscrits dans la définition même de la nation. Déjà, en 1849 , l'idée de germanité (Deutschtum) qui comprend également, en principe, les Allemands vivant dans des États voisins à cause de leur appartenance au peuple allemand, était à l'origine des débats sur la Constitution qui cherchait à faire de la nationalité une spécificité allemande et des droits fondamentaux du "peuple allemand", qui de fait sont des citoyens allemands '. Ainsi se trouvent combinées culture et politique, une identité culturelle qui constitue la base d'une identité politique.

Le mouvement des idées nationalistes date de la période révolutionnaire et de l'État allemand unifié de 1871. L'idée de nation, d'abord culturelle, a donc précédé la naissance de l'État et la conscience nationale s'est élaborée avant l'organisation politique. La nationalité fondée sur le droit du sang avait déjà posé un problème conceptuel et pratique à sa création, dans la mesure où les nouvelles frontières de l'Empire favorisaient un croisement des concepts de peuple et de territoire. La petite Allemagne excluait de la nation les étrangers des États voisins et incluait en principe les Allemands ethniques qui vivaient en dehors de son territoire. Par ailleurs, l'absence de centre qui se traduit par la faiblesse des institutions n'a pas produit une identification politique, mais a renforcé un sens d'appartenance ethnoculturelle (Volksgemeinschaft) fondée sur le principe d'ascendance. Comme le rap-

1. Cf. D. Gosewinkel, - Citizenship and Nationhood : The Historical Development of the German Case ", dans U.K. Preuss, F. Requejo (dir.), European Citizenship, Multiculturalism and the State, Baden-Baden, Nomos, Verl.-Ges., 1998, p. 125-135. pelle Rogers Brubaker, il en a résulté deux formes de citoyenneté : l'une politique, correspondant au territoire, l'autre spirituelle et ethnique, fondée sur les ancêtres communs ${ }^{2}$. C'est ainsi qu'une loi adoptée en 1913 (en vigueur jusqu'en janvier 2000), " sur l'appartenance à l'État et à l'Empire ", a soutenu l'aspect ethnique de la citoyenneté, en permettant aux Allemands vivant en dehors des frontières de garder la nationalité allemande et en rejetant la possibilité d'accorder la nationalité allemande aux étrangers nés à l'intérieur du pays.

L'identité allemande qui dépassait par conséquent les frontières de l'Empire, n'avait donc pas un sens politique reconnu dans le nouvel État. Cela n'a pas empêché pour autant de définir la nationalité en combinant les ancêtres et le territoire (du passé), de sorte que les Allemands en dehors des frontières de l'État garderaient au moins leur nationalité allemande. En 1949, la Loi fondamentale a confirmé le principe de la citoyenneté liée à l'appartenance au peuple allemand. L'article $116 \mathrm{de}$ ladite Loi considère comme allemande toute personne d'ascendance allemande vivant à l'intérieur des frontières de l'Empire.

Une telle représentation de la nation rencontre le droit, notamment dans le cas des Aussiedler. Leur naturalisation de droit sur preuve d'appartenance au peuple allemand dès l'arrivée renvoie à la confrontation entre territoire (toujours du passé), culture et identité. Culture et identité sont pourtant des concepts dynamiques qui varient dans le temps et en fonction des interactions. Par conséquent, aujourd'hui, leur usage, en référence au passé crée d'emblée une ambiguité par rapport à la réalité sociale comme le prouvent d'ailleurs les problèmes d'intégration culturelle et sociales des Aussiedler. Plusieurs études montrent leur difficulté d'adaptation, leur degré d'intégration et leur perception sociale et culturelle dans la société alle-

2. W.R. Brubaker, Citizenship..., op. cit. 
mande. Malgré la déclaration d'appartenance aux mêmes ancêtres, les Aussiedler seraient plus étrangers de langues et de culture, notamment politique, que les populations issues de l'immigration, souvent nées sur le territoire allemand ou du moins socialisées dans les institutions nationales. Dans le langage sociologique, ils semblent même constituer une nouvelle catégorie sociale qui renvoie les Allemands du territoire de l'État-nation à l'appellation Einheimische ou encore Bundesdeutsche (Allemands de souche), pour les distinguer des Allemands " hors territoire " 1 .

\section{O NATION ET IMMIGRATION}

Une telle représentation de la nation a conduit aussi à un discours officiel qui rappelait à chaque occasion et jusqu'à récemment que "l'Allemagne n'est pas un pays d'immigration". Or plusieurs recherches s'accordent maintenant à démontrer que "l'Allemagne est un pays d'immigration" ".

L'immigration en Allemagne est surtout le résultat d'une politique de l'emploi. À la fin du $19^{\circ}$ siècle, l'industrialisation, ou plutôt la transition " d'un État agraire avec une forte industrie à un État industriel avec une base agraire importante ", avait provoqué d'abord une immigration interne de l'Est vers l'Ouest '. Il s'agissait dans la plupart des cas de Polonais allemands. Mais vers la fin du siècle, les Polonais étrangers de Russie ou d'Autriche dépassaient en nombre, et de loin, les Polonais allemands

1. B. Dietz, "Jugendlilsche Aussiedler in Deutschland Risiken und Chancen der Integration ", dans K.J. Bade, J. Oltmer (dir.), Aussiedler: deutsche Einwanderer aus Osteuropa, IMIS-Scriften 8. Osnabrück, Universitätverlag Rasch, 1999, p. 153-176.

2. Cf. R. Münz. W. Seifer, R. Ulrich, Zuwavderung nach Deutschland. Strikturen, Wirungen, Perspektiven. Frankfort am Main, Campus, 1997.

3. Cf. les travaux de K.J. Bade (dir.) Bevölkerung, Arbeitsmarket und Wanderung in Deutsch seit der Mitte des 19 juts. tome 1. Ostfilden, Scripta Mercaturae Verlag, 1984 ; - Trends and Issues of Historical Migration Research in the Federal Republic of Germany * Migration 6, 1989, p. 7-28: * Politik in der Einwanderungssituation: Migration - Integration Minderheiten * dans K.J. Bade, Deutsche im Ausland Fremde in Deutscbland - Migration in Geschicbte und Gegenwart, München, Verlag C.H. Beck, 1994, p. 442-455. dans l'agriculture à l'Est. La Silésie et la Russie orientale manquant de maind'œuvre, les responsables commencèrent alors à se préoccuper de la " polonisation " de l'Ouest, c'est-à-dire du contact des Polonais allemands qui y travaillaient avec des Polonais étrangers. Le travail saisonnier apparaissait dès lors comme la seule possibilité de combiner les intérêts économiques et la protection de l'envahissement des Polonais étrangers (Überfremdung). Ainsi s'est défini un système de rotation fondé sur le contrôle du respect de la période de travail (Karenzzeit) des Polonais étrangers à l'Ouest. Mais parallèlement le besoin permanent d'une main-d'œuvre bon marché suscité par l'industrialisation donna lieu à ce que l'on a appelé le recours à "l'armée de réserve industrielle ", formée de Hollandais, d'Autrichiens et surtout d'Italiens, qui a changé la structure de la population étrangère. Après la seconde guerre mondiale, l'appel à la maind'œuvre étrangère devient indispensable pour la reconstruction économique, s'agissant cette fois de population en provenance de l'Est de la Méditerranée, de la Grèce, de l'ex-Yougoslavie, de la Turquie.

Aujourd'hui presque 7 millions d'étrangers résident en Allemagne, dont 2,5 millions de nationalité turque. Leur présence remonte aux années 1960, présence jusqu'à une période assez récente " assimilée "à un séjour temporaire avec un espoir fondé sur le discours qui rejette la réalité de l'immigration et encourage le retour. C'est sur ces discours que se sont appuyés plus tard, dans les années 1980, les politiques dites d'étrangers (Ausländerpolitik) ou de projet de loi sur la citoyenneté appelée loi pour les étrangers (Ausländergesetz). Ces discours traduisaient en réalité une volonté de maintenir les Ausländer dans un statut d'étrangers, à l'écart de la nation et de la citoyenneté, sans les considérer comme futurs citoyens ni les inclure dans la communauté nationale. Quant à la naturalisation, elle ne peut être conçue que comme l'ultime étape de l'assimilation. 
En même temps, dans les années 1980 , l'Allemagne, comme la France, se définit davantage comme " multiculturelle". Il se crée même à la municipalité de Francfort un secteur des "Affaires multiculturelles ", dont le responsable, en même temps maire-adjoint de la ville, Daniel CohnBendit, prône une " démocratie multiculturelle " qui s'inspirerait du contrat social de Rousseau '. Mais l'usage du terme a comme fonction un constat et une stratégie de représentation d'une société où coexistent des populations avec des nationalités et religions différentes. Dans un souci de société démocratique où règnent les principes de l'égalité de droit, ces discours ont comme objectif de faire accepter à l'opinion publique la diversité anthropologique comme un fait inhérent à toute société moderne. Cela apparaît, de la part des militants et des porte-parole des partis d'opposition, comme une façon de faire prendre conscience à l'opinion publique et à la classe politique en général que les étrangers "sont là pour rester "; que l'Allemagne est en fait un pays d'immigration et de fait une société multiculturelle. De Gastarbeiter (travailleurs invités), ils deviennent Ausländer (des étrangers) et, dans les années 1970, des ausländische Arbeiter (des travailleurs étrangers) ou parfois des Einwanderer (immigrés), et non plus simplement des Gäste (des invités), mais immigrés, ou minorités, ou les deux, Einwanderungsminorität) (minorité immigrée) ou encore ausländische Mitbürger (des concitoyens étrangers), des citoyens sans l'être.

À travers cette terminologie, malgré le changement, transparaît la perception de l'étranger et le lien entre les structures institutionnelles et les structures mentales ainsi que la relation entre la rhétorique et le juridique, fondement du débat sur la citoyenneté. En effet, l'immigration dans les années 1960 , comme au début du siècle, renvoie à

1. Ses ambitions et ses intentions sont développées dans un ouvrage qu'il a écrit en $1992: D$. Cohn-Bendit, T. Schmid, Heimat Babylon. Das Wagnis der Multikulturellen Demokratie, Hambourg, Hoffman and Campe, 1992. "l'importation de travailleurs " ${ }^{2}$. La présence étrangère depuis cette période-là ne change en rien les lois sur " la germanité " et sur la nationalité allemande. Cette dernière, bien que contestée en tant qu'entrave aux principes démocratiques, apparaît cohérente avec la définition qu'elle donne de la nation allemande. En effet, si celle-ci se définit par une culture commune, le principe de base de la nationalité demeure fondé sur l'appartenance aux ancêtres communs transmise par le droit du sang (jus sanguinis). Mais en 1993 une nouvelle loi sur les étrangers (Ausländengesetz) a été élaborée pour faciliter l'intégration des étrangers comme une réaction aux flux d'immigration de l'Est. Elle s'est imposée comme une " obligation morale " d'inclusion des populations étrangères installées sur le sol allemand depuis trois générations. Cette loi a introduit pour la première fois le critère de socialisation pour les petits-enfants des Gastarbeiter, candidats à la naturalisation. D'après cette loi, un jeune étranger peut obtenir la naturalisation de droit s'il en présente la demande entre 16 ans et 23 ans, s'il réside en RFA depuis huit ans de façon régulière, s'il a fréquenté un établissement scolaire depuis six ans, dont au moins quatre ans dans un établissement d'enseignement général, et enfin s'il n'a pas commis de délit. Par ailleurs, le prix de la naturalisation, qui variait entre 3000 et $5000 \mathrm{DM}$, a été abaissé à $100 \mathrm{DM}$ pour ces jeunes. Le nombre d'étrangers naturalisés, qui variait entre 20000 et 30000 par an entre 1973 et 1989, atteint les 101377 en 1990. En 1993, plus de 29000 étrangers ont accédé à la citoyenneté allemande par régularisation et 44900 par naturalisation sur décisions discrétionnaires des autorités allemandes ${ }^{3}$.

\section{K.J. Bade, op. cit.}

3. En 1993, le nombre des demandes de naturalisation atteint 44950 , et continue d'augmenter depuis. Cf. P. Schmidt, $\mathrm{S}$. Weick, "Intégration sociale des étrangers en Allemagne :, Revue de l'OFCE, 69, avril 1999, p. 267-276. Cf. aussi R. Münz, R. Ulrich, * Germany and its immigrants : a sociodemographic analysis *, Journal of Ethnic and Migration Studies, janvier $1998,24,1$, p. 25-56. 
En 1994, une mesure annoncée par le gouvernement de coalition (CDU-FDP) concerne la troisième génération d'enfants d'origine turque. La discussion prend la forme de ce que les responsables associatifs appellent "la citoyenneté enfantine " (Kindesstaatsangebörigkeit). L'objectif d'un tel projet de loi est d'abord d'accorder la nationalité allemande à l'enfant né de parents étrangers, dès sa naissance si l'un de ses parents étrangers est lui-même né en République fédérale. Cela introduit en somme un " double droit du sol " déjà traditionnellement reconnu en France, mais s'assortit cependant de cette restriction que le couple marié doit fournir la preuve de sa résidence en Allemagne pendant au moins dix ans avant la naissance de l'enfant. De plus, à 18 ans l'enfant peut revenir en arrière et faire son choix entre la nationalité allemande et celle de ses parents. Il a été question aussi de leur accorder une "garantie de naturalisation" (Einbürgerungszusicberung), une proposition qui serait clairement mentionnée sur la pièce d'identité à leur naissance '. Cela sans oublier que le choix d'une nationalité unique s'impose en Allemagne du fait de l'article relatif à la renonciation de la nationalité d'origine dans la Constitution.

\section{O PARTICIPATION ET CITOYENNETÉ}

Bien entendu les lois qui régissent la citoyenneté affectent les modes de participation et les stratégies des acteurs. L'absence des droits politiques que procure la citoyenneté légale conduit l'étranger à développer des stratégies de compensation. Cela n'exclut pas l'intégration, bien au contraire, mais conduit à chercher des voies indirectes pour y aboutir ; le citoyen n'est plus un " spectateur qui vote "comme dit Rousseau, mais un acteur qui cherche à faire voter par son influence sur l'opinion publique ou les décisions gouvernementales.

1. La Lettre de la citoyenneté, 31, janvier-février 1998.
La demande de reconnaissance des spécificités identitaires ont conduit les " étrangers " à agir dans l'espace public et à manifester ainsi leur engagement et leur appartenance au moins de fait à une communauté politique. Cet engagement se traduit avec une forte participation qui s'opère surtout dans le cadre des associations reconnues par les pouvoirs publics, dans les activités des communautés locales - culturelles ou ethniques -, dans l'expansion économique, bref dans la société civile dans son ensemble. C'est là que s'exerce la citoyenneté. Il s'agit d'une forme de citoyenneté qui dérive surtout de sa pratique sociale. Elle est l'expression de l'engagement de l'individu vis-à-vis du bien commun que représente la communauté nationale ${ }^{2}$.

En effet, depuis les années 1980, la prolifération des associations dites d'immigrés s'inscrit dans les "politiques pour les étrangers" (Ausländerpolitik), qui visent leur intégration, aussi contradictoire que cela puisse paraître dans les termes. Dans cette perspective, l'administration allemande encourage les étrangers à s'auto-organiser (Selbsthilfe) par le biais des associations. L'idée est de voir les étrangers créer leurs propres organismes pour combattre la délinquance, la pauvreté, la criminalité. La procédure rappelle le libéralisme américain où les associations volontaires issues des communautés ethniques elles-mêmes sont en même temps des institutions d'entraide ou de bienfaisance au service de leurs membres, soucieuses aussi de gérer les problèmes sociaux qui les touchent plus particulièrement, à la différence fondamentale du statut du citoyen des membres d'une communauté ethnique aux États-Unis. En Allemagne, cette logique de communauté ethnique, structurée et intégrée, se prenant en charge, s'accompagne parallèlement de

2. Pour citoyenneté comme sentiment d'appartenance et citoyenneté comme engagement, of. J. Leca, - Individualisme et citoyenneté ", dans P. Birnbaum, J. Leca (dir.), Sur l'individualisme, Paris, Presses de Sciences Po, 1986, p. 159213. 
la lutte pour l'égalité des droits inscrite dans la définition même de la citoyenneté. Elle se définit par rapport à la société civile dans laquelle prennent forme les organisations et les actions politiques. Elle traduit dans les faits l'extension du système corporatiste qui caractérise la société civile des non-citoyens, sur la base de la résidence et au regard d'une égalité de droits qui correspond à une forme de citoyenneté. Elle traduit aussi la volonté d'intégration des Turcs, organisés désormais en communauté intégrée dans la structure sociale de la République fédérale selon une logique de portée générale où les institutions para-publiques ont leur place dans la société civile et jouent un rôle important dans les décisions politiques ${ }^{1}$.

$\mathrm{Au}$ niveau local, leur engagement s'est concrétisé par la mise en place des structures telles que des Conseils consultatifs pour étrangers (Ausländerbeiräte) créés vers le milieu des années 1970 , ou des "Commissions extramunicipales des étrangers " ${ }^{2}$. Ces organisations permettent aux "non-nationaux ", selon la terminologie officielle en République fédérale, de s'engager pour le "bien commun", ici la ville ou la cité. Les étrangers organisés dans de telles structures cherchent à représenter auprès de la municipalité des intérêts communs, concernant en particulier les écoles, les crèches, les espaces verts ou les commerces. L'établissement des listes autonomes de candidature à ces instances suscite une forte concurrence et occasionne parfois des tensions interethnique dans la ville concernée tout comme au sein des associations ou entre elles.

1. C'est ce qui conduit P. Katzenstein à se référer à l'État allemand comme un - État semi-souverain : : P. J. Katzenstein, Policy and Politics in West Germany. The Growth of a Semi Sovereign State, Philadelphie, Temple University Press, 1987, p. 168-192. Cf. aussi S. Berman. "Civil Society and Political Institutionalization ", American Behavioral Scientist, 40 (5), mars-avril 1997, p. 562-574.

2. La Cour constitutionnelle a rejeté en 1989 le droit de vote local aux étrangers que le Parlement de Hambourg avait accordé en 1987, au motif, comme en France (article 3 de la Constitution française), que seule la possession de la nationalité allemande pouvait donner accès au droit de vote local ou fédéral. Ce qui a maintenu les étrangers en dehors de la participation électorale.
Toutefois, la portée purement symbolique des attributions de ces conseils empêche leurs membres élus d'exercer une quelconque influence sur les décisions communales. En fait, ils se contentent de soumettre aux autorités locales les problèmes auxquels font face les commerçants ou les étudiants étrangers, ou encore, par exemple, de présenter des rapports qui font état du dysfonctionnement des bureaux d'accueil. L'intérêt porté à leur élection est pourtant massif. À Bamberg, par exemple, à l'automne 1994, le taux de participation des étrangers à la désignation du conseil consultatif s'est élevé à $48,5 \%$ en général, et à $78 \%$ chez les Turcs dont les candidats ont obtenu quatre sièges ${ }^{3}$.

Outre les effets des associations et des mobilisations locales sur l'opinion publique, l'intégration dans la société civile s'opère aussi par une réussite économique comme preuve de citoyenneté ${ }^{4}$. Un rapport publié à Bruxelles en 1991 estime à environ 57 milliards de DM l'apport économique direct ou indirect des Turcs, somme qui dépasse de loin les dépenses de l'État providence pour les étrangers, qui n'atteignent que 16 milliards de DM ${ }^{5}$. Les associations des hommes d'affaires turcs recensent, pour 1992, 35000 entrepreneurs, allant du restaurateur à l'industriel, qui emploient au total 150000 Turcs et 75000 Allemands. Leur chiffre d'affaires annuel s'élève à 25 milliards de DM et ils ont payé, en 1991, 1 milliard de DM d'impôts ". Ces acteurs économiques jouent non seulement un rôle

3. Cf. L. Yalçin-Heckmann, " The Perils of Ethnic Associational Life in Europe : Turkish Migrants In Germany and France ", Paper submitted to the Workshop - Culture, Communication and Discourse : Negotiating Difference in MultiFthnic Alliances . organisé par ICCCR, Universities of Manchester and KEFLE, 9-12 décembre 1995.

4. M. Walzer, "The Civil Society Argument", dans C. Mouffe (ed.), Radical Democracy, London, Verso, 1994.

5. * Migrations New Sheet *, Bruxelles, décembre 1991, cité par The Economic and Political Impact of Turkish Migration in Germany, Zentrum für Türkeistuden, mars 1993.

6. Statistiques de l'Union des entrepreneurs turcs de Berlin. Cf. aussi, Konsumgewobnbeiten und wirtschaftiche situation der türkiscben Bevölkerung in der Bundesrepublik Deutschland, Essen, Zentrum für Türkeistuden, septembre 1992. 
important dans les relations entre l'Allemagne et la Turquie, mais tiennent en plus entre leurs mains le sort des projets d'investissements allemands dans les républiques turcophones de l'ancienne Union Soviétique. Régulièrement les statistiques font état de leur investissement, les médias attirent l'attention sur leur consommation et les entreprises allemandes expriment leur solidarité civile et cherchent ainsi à agir sur les politiques les concernant.

Mais la sécurité fondée sur la présence économique peut-elle toutefois compenser l'insécurité politique par manque de droit de protection? Une intégration économique peut-elle ouvrir la voie aux droits politiques? L'histoire apporte une réponse partielle à cette question dans la mesure où, au $19^{e}$ siècle, le milieu économique avait su intégrer l'Allemagne dans la compétition économique internationale pendant la grande dépression de 1873-1896. C'est sur cette base qu'elle acquit ensuite une importance et fit sentir son poids sur les décisions politiques du pays. En vertu de la même logique, des secteurs d'activité comme l'organisation des médecins turcs depuis 1990, puis dernièrement les associations d'hommes d'affaires de différents Länder se préparent désormais à agir comme groupes de pression capables de négocier les intérêts collectifs allant de la protection des droits sociaux et culturels aux mesures à prendre contre la xénophobie. S'appuyant du moins dans le discours sur le rôle joué par le facteur économique dans la redéfinition de l'identité allemande après la guerre, ils se fondent également sur leur réussite dans ce domaine pour se transformer en force politique.

Faut-il voir dans la réussite économique une façon de s'approprier le "miracle économique ", dans l'espoir que la prospérité et le bien-être qu'il apporte donnent un nouveau contenu à l'identité du pays audelà de la vieille référence ethnique ? Ou bien cette approche tient-elle lieu d'interprétation de la "citoyenneté ", dans un sens non moins traditionnel, lié au statut d'origine médiévale de bourgeois (Bürger)? En Allemagne, cette seconde vision de la citoyenneté se rapporte plus à l'appartenance à la société civile qu'à l'allégeance à la communauté politique, la société civile y étant définie comme une société de bourgeois (die bürgerlische Gesellschaft) qui non seulement se distingue de l'État et de ses institutions mais s'oppose à eux. Dans ce sens, tout individu qui participe à la vie publique dominée par la compétition économique peut être considéré comme citoyen. "Cela suggère que le citoyen est synonyme de bourgeois, les deux faces de la même monnaie " écrit Ralf Dahrendorf ". C'est dans cette logique qu'il faudrait peutêtre interpréter le terme de ausländische Mitbürger (concitoyens étrangers) introduit dans le débat public par les Verts, la formule revenant à accepter les ressortissants turcs dans la société allemande par le biais d'une citoyenneté économique faute de citoyenneté politique. Leur " embourgeoisement "serait-il dans ce cas un pas vers la naturalisation, comme le laisse entendre le terme allemand Einbürgerung même s'il est utilisé en référence à l'État (Staatsbürger) et non pas à la cité comme c'est le cas du bourgeois ? Cette conception très spécifique de la citoyenneté met l'économie au service du politique et donne un poids politique aux Turcs en $\mathrm{Al}$ lemagne qui sont du coup citoyens sans l'être, dans la mesure où ils ne font pas partie de la catégorie de "citoyens à part entière ou citoyens liés par le sang ", déclaré par la Constitution ${ }^{2}$.

$\mathrm{Au}$ lendemain de la seconde guerre mondiale, avec T.H. Marshall, la citoyenneté s'est trouvée reconsidérée en termes de classe sociale, en faisant glisser le

1. R. Dahrendorf, The Modern Social Conflict. An Essay on the Politics of Liberty, Berkeley, University of California Press, 1988, p. 34.

2. D. Gosewinkel, . Citizenship and Nationhood... ", cité. Lauteur souligne que la Constitution démocratique de Weimar (1919) avait mis l'accent sur une conception de société civile avec des droits égaux pour hommes et femmes, et coincidait avec le statut légal de la citoyenneté définie comme affiliation avec l'État. Cette logique fut détruite en 1935 par la loi de Nuremberg. 
concept de son contenu légal et politique à une approche sociale de l'égalité devant le droit ${ }^{1}$. Mais une telle conception de la citoyenneté inscrit les droits sociaux dans la continuité des droits politiques. Si donc une "citoyenneté sociale" qui inclut l'étranger dans les structures corporatistes existantes se traduit à ce niveau par une participation directe dans la société civile ce que Habermas appelle une " citoyenneté passive "-, une citoyenneté trouve sa légitimité dans le développement de l'État providence avec accès égal aux avantages sociaux et même protection par la Constitution dans le domaine des Droits de l'homme que les nationaux. Cette participation n'est qu'indirecte en ce qui concerne la citoyenneté proprement politique (ou "active "). Seule la citoyenneté légale et politique ouvre un droit de participation à la communauté politique au sens plein et le passage s'effectue dans leur cas par la "naturalisation" qui considère la durée de séjour, l'apport en travail et en service, la conformité aux normes sociales. D'après Joseph Carens, une des conditions les plus pertinentes pour la naturalisation est justifiée par l'aspiration des individus en relation avec les attentes de la société nationale ${ }^{2}$.

\section{○ DOUBLE NATIONALITÉ OU MINORITÉ NATIONALE}

Depuis 1990 , les débats sur la citoyenneté en Allemagne portent sur la double nationalité. Le maintien de la nationalité d'origine devient une manière de négocier la citoyenneté. Ces négociations reprennent avec chaque gouvernement. La nouvelle coalition (SPD et Verts) propose le

1. T.H. Marshall, Class, Citizenship and Social Development, Chicago, The University of Chicago Press, 1964 (1'édition). L'auteur a attiré l'attention sur l'extension des pratiques sociales de la citoyenneté à d'autres domaines tels que la santé, l'éducation, l'accès aux avantages sociaux en général.

2. Cf. J.H. Carens, - Why Naturalization Should be Easy? , dans N.M.J. Pickus, Immigration and Citizenship in

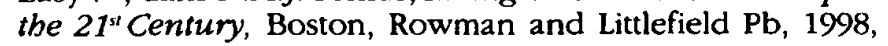
p. 141-149. maintien de la nationalité antérieure pour les Turcs comme condition d'accès à la nationalité, c'est-à-dire l'abandon de l'article relatif à la renonciation. Ce projet a situé la double nationalité au cour du débat sur la citoyenneté, sur son lien avec l'identité nationale et sur le respect de la Constitution, conduisant l'opposition (CDU et CSU) à une campagne de signatures contre elle, et les acteurs politiques issus de l'immigration turque à réclamer la double nationalité comme condition d'accès à la citoyenneté allemande. La loi de janvier 2000 d'après laquelle l'enfant d'étranger acquiert la nationalité allemande à sa naissance accorde le maintien de la nationalité des parents. Mais entre 16 ans et 23 ans, l'enfant devrait choisir entre les deux nationalités : rester allemand ou devenir " étranger ".

La double nationalité remet en cause le lien entre citoyenneté, nationalité et identité. Dans le cas des Turcs, elle trouverait son fondement dans une logique qui renverrait l'identité à la nationalité de l'État-nation d'origine et la citoyenneté à un droit dans le pays de résidence : droit de séjour permanent, droit de protection contre le racisme et droit à l'égalité dans le domaine politique. La citoyenneté devient ainsi un moyen de garantir l'installation sur le territoire et non d'assurer une intégration culturelle. Dans cette perspective, la citoyenneté n'est qu'un simple statut juridique et la nationalité se définit uniquement par son aspect ethnique, religieux et/ou culturel. C'est la logique même de construction de minorité ${ }^{3}$ : une "minorité " qui, dans le cas des Turcs, repose à la fois sur une identité nationale turque et une identité religieuse musulmane. La minorité nationale se définirait par son statut juridique d'étranger, et la minorité religieuse par l'absence d'une reconnaissance institutionnelle de l'islam par rapport aux

3. Le concept de "minorité " dans le contexte allemand, est réservé aux Allemands eux-mêmes en situation de minorité à la suite de leur exil pendant la deuxième guerre mondiale. D'où le rejet du même concept en référence aux populations immigrées. 
autres religions qui jouissent d'un statut officiel car ils relèvent d'un droit public.

La question se pose de savoir quel est le rôle des États dans la construction de minorité ? Les politiques d'intégration appelées Ausländerpolitik répondent en partie à cette question. En effet, les pouvoirs publics allemands manifestent leur souhait de voir les Turcs s'organiser en communauté ethnique unifiée autour d'intérêts communs qui dépassent leurs clivages idéologiques, religieux, ethniques et linguistiques. L'objectif déclaré de la Commission des étrangers (Ausländerberauftragte) est d'aider les populations issues de l'immigration, notamment les Turcs, à former des organisations représentatives d'une "communauté où règne le consensus "plutôt que de voir une multitude de groupes formels ou informels en conflit. Même si, pour les pouvoirs publics, il est question d'agir contre le cloisonnement ou la marginalisation réelle des étrangers dans le domaine social, culturel, économique, politique, de les aider à se rassembler en communautés unifiées et solidaires à l'exemple des Églises, cela devient pour les étrangers la seule façon d'obtenir une légitimité et de faire " reconnaittre leur présence permanente " en Allemagne.

Dans cette perspective, les militants associatifs reprennent à leur compte les discours des pouvoirs publics, suivent leurs consignes pour organiser ou structurer une telle communauté. Ils rassemblent leurs forces pour créer des associations-toit (Dachorganization), c'est-à-dire des fédérations d'associations dans chaque Land, voire au niveau fédéral, en vue de regrouper l'ensemble des ressortissants turcs ${ }^{1}$. Ce que

1. Comme, par exemple, la türkische Gemeinde zu Berlin. L'exemple est pris sur la judische Gemeinde zu Berlin à la fois dans le discours et dans les actions, comme si les militants voulaient afficher les bonnes relations entre la Turquie et Israël. Mais l'exemple est surtout pris comme un moyen pour eux de montrer leur ouverture vis-à-vis des autres communautés ou minorités. Plus qu'une ouverture, c'est comme si l'association voulait prouver à l'opinion allemande aussi bien qu'à ses propres adhérents les efforts dans la formation d'une - minorité - à l'exemple d'une " minorité modèle "et les sensibiliser à une lutte commune contre le racisme que le soutien des organisations juives ne peut que renforcer. les pouvoirs publics appellent communauté ethnique trouve un écho transformé dans l'idée de minorité ethnique chez les dirigeants d'associations ou chez les militants actifs dans l'immigration turque en Allemagne. Ils entendent désigner, par ce terme, la présence structurelle d'une population culturellement différente comptant presque 2 millions d'individus de toutes catégories sociales (chômeurs, ouvriers qualifiés ou non, étudiants, cadres, commerçants, industriels) qui tous expriment leur appartenance à des cultures régionales, linguistiques ou ethniques différentes : une türkische Minderbeit, comme le suggèrent les partisans du SPD dans les débats politiques, en soulignant la nationalité d'origine comme le fondement de la construction d'une minorité. On peut se demander aussi si ce n'est pas le rappel permanent d'une identité nationale allemande définie par l'ascendance qui conduit les non-Allemands à se considérer de la même façon, c'est-à-dire à se définir à partir des mêmes critères ethniques que serait la nationalité antérieure. Ou bien si ce sont l'environnement politique et le statut juridique qui maintiennent la différence des nationalités comme élément constitutif de la construction d'une identité collective à revendiquer, voire de son institutionnalisation en tant que minorité.

L'État turc contribue autant que l'État allemand à la construction d'une minorité ethnique à base de nationalité en Allemagne. Il cherche, à travers l'existence d'une telle structure, la constitution d'un "lobby " turc en Allemagne destiné à défendre les intérêts et l'image de la Turquie à la fois en Allemagne et en Europe, notamment auprès des institutions supranationales. Ces stratégies distinctes se retrouvent plus dans les représentations que dans les modes d'organisation. En effet, communauté ethnique, minorité nationale ou lobby turc se réfèrent tous trois à une structure centrée sur la nationalité avec des intérêts identitaires similaires. Ne faut-il pas voir là aussi l'écho du désir exprimé 
par les gouvernements successifs de voir les "invités " retourner un jour dans leur pays, les renvoyant ainsi à "leur État " comme seul refuge et source de sécurité et de droit, comme pour leur rappeler le principe d'accès à la citoyenneté allemande fondée principalement sur une appartenance culturelle commune?

Il n'empêche que la minorité se définit surtout à travers son lien avec l'État allemand, seul cadre légitime qui définit les limites de la reconnaissance. Les stratégies de compensation par l'engagement dans les associations ou une intégration dans la société civile font preuve d'une volonté d'intégration dans la société politique. Cette opération s'opère à travers les structures communautaires mises à leur disposition. Ce sont ces mêmes structures qui paradoxalement les conduisent à se replier sur eux-mêmes et à exprimer leur attachement à une nationalité d'origine et leur lutte pour sa représentation. La demande de la double nationalité se trouve fondée précisément dans cette contradiction des politiques dites pour étrangers (Ausländerpolitik): la reconnaissance des communautés d'appartenance inscrites dans les structures de la société. Par conséquent, la citoyenneté comme engagement se limite de la sorte au groupe restreint, sans accès à la communauté politique globale et sans lien non plus avec la communauté de voisinage et les autres populations étrangères. Mais, en même temps, que la pratique de la citoyenneté soit politique, juridique, sociale, économique, et son contenu identitaire, culturel ou légal, cette combinaison se résume en un sentiment de loyauté dirigé simultanément vers le groupe, la communauté, la société civile et l'État. C'est dans leur interpénétration que se profilent les stratégies des acteurs et la revendication de la double nationalité.

Par ailleurs, le langage politique attribue une terminologie qui sépare la citoyenneté de la nationalité et de l'identité. La citoyenneté (Staatsbürgerschaft) ou la nationalité (Staatsangehörigkeit) se réfèrent toutes deux à l'État, mais la première comme appareil, la deuxième comme appartenance. La référence à la double nationalité (die doppelte Staatsbürgerschaft) trouve son fondement dans cette dualité en réalité complémentaire : la construction d'une minorité ethnique (nationale) en Allemagne avec une identité de citoyen qui met en évidence une "assimilation " politique et institutionnelle. Cette analyse s'oppose aux thèses pessimistes qui soulignent le " retard dans l'assimilation ", que pourrait causer le maintien de la nationalité du pays d'origine ${ }^{~}$. Au contraire, la double nationalité revendiquée par les Turcs pourrait être perçue comme un droit qui permettrait précisément de négocier une personnalité morale ethno-nationale construite sur une référence triple : à la société civile allemande par la résidence, avec les droits et les devoirs civils qui lui sont liés et à la nationalité turque - pour ceux qui s'y reconnaissent - au plan de l'identité, et ce qui conduit au renforcement d'une double appartenance communautaire, et, à l'État allemand. C'est cette multiplicité qui devient l'enjeu de la citoyenneté à négocier.

La volonté d'intégration dans la communauté politique par la demande de la citoyenneté et par un "attachement "à la nationalité d'origine pourrait être perçue pour les groupes qui affichent une identité spécifique comme un moyen de sortir de leur marginalité politique. Elle traduit par conséquent une lutte pour l'émancipation. Mais, contrairement à l'émancipation des Lumières qui sépare la religion de la vie publique et l'individu de sa communauté pour assurer son identification primordiale avec la communauté nationale, la double nationalité pourrait trouver un fondement dans la volonté de participation avec des droits égaux qui serait reconnue à des identités religieuses ou communautaires à inscrire dans le cadre des structures de l'État.

1. Argument développé par P. H. Schuck, * Plural Citizenship - dans N. M. J. Pickus, op. cit., p. 149-193. 
Mais les distinctions terminologiques et conceptuelles en référence à l'État, sources d'ambiguités, trouvent un écho dans les expressions qui hésitent sur la façon de nommer ces étrangers qui ont pourtant pris racine sur le territoire national : des "non-Allemands" pour la droite libérale, des " concitoyens étrangers " pour les Verts qui veulent sensibiliser l'opinion générale sur leur apport à la société, et enfin des Türken mit Deutsche Pass (Turcs avec un passeport allemand) pour désigner tous ensemble les plus de 250000 personnes originaires de Turquie naturalisées allemandes. Ces derniers constituent déjà un potentiel électoral important et les partis rivalisent pour définir la place à leur accorder dans la communauté politique. Une étude réalisée en 1994 sur le comportement électoral qu'auraient eu les Turcs s'ils avaient été citoyens allemands a révélé que $49 \%$ auraient voté pour le SPD (alors qu'ils étaient $67 \%$ en 1986), $11 \%$ pour les Verts, $10 \%$ pour le FDP, $6 \%$ pour la CDU. Bien que le nombre des électeurs citoyens allemands d'origine turque, âgés de 18 ans et plus, ne dépasse pas 35000 individus, les partis publient de la propagande dans les journaux communautaires comme s'ils voulaient réaliser une sorte d'investissement électoral. Le FDP aimerait miser sur le vote des Turcs pour dépasser le seuil de $5 \%$ en dessous duquel il ne peut avoir d'élus ; le SPD soutient leurs grandes associations de peur de voir les militants de celles-ci adhérer à d'autres partis '.

Ces expressions et ces approches mettent en évidence le lien, ou l'absence de lien, que certains voudraient créer ou creuser entre citoyens et nationaux. Séparer l'électeur sur la base de la nationalité - d'origine ou double - met en évidence la difficulté de l'opinion publique et de la

1. F. Sen, Y. Karakasoglu. $f$. Almanya da jasayan Tiurklerin ce diger yabancilarin seçme te seçilme hakki. partiler te fifte iatandaslik ïzerine gërnisleri. Essen. Zentrum für Türkeistudien. septembre 1994 (Opinion des Turcs et autres étrangers sur le droit de vote. les partis. la double nationalité en République fédérale allemande). classe politique à accepter les " étrangers " comme faisant partie de la nation. Au lieu de limiter à la société civile l'identification à la communauté ethnique, une telle approche qui donne son fondement à la double nationalité installe la dualité entre minorité et citoyenneté dans la communauté politique.

\section{O VERS UNE NOUVELLE UNITÉ ?}

La vraie question ne peut donc être que la citoyenneté et non la double nationalité. Alors que cette dernière invite à un réexamen de la conception de l'État et de son rapport à la définition de la nation, la citoyenneté invite l'individu à faire partie de la communauté nationale. De même, la double nationalité n'implique pas une participation simultanément dans deux espaces politiques; un citoyen n'est le citoyen que d'un État où il exerce pleinement ses droits et ses devoirs. La naturalisation, processus par lequel l'individu accède à l'adhésion à une communauté politique assure son inclusion, sous certaines conditions certes, mais ces conditions ne concernent que le lien entre l'individu et sa nouvelle communauté politique dans le cadre de son État, donc territorialement limitée. Bien entendu, cela ne peut s'accompagner ni d'un oubli ni d'une amnésie culturelle d'appartenance par rapport à une citoyenneté ou nationalité antérieure, mais cette dernière ne constitue plus qu'un document réservé à la vie privée. Quant à sa validité juridique, elle relève tout simplement d'ententes entre États. Par conséquent, l'argument développé autour de "l'influence démocratique " 2 de la double nationalité, c'est-à-dire de l'utilisation dans le pays d'origine des valeurs constitutionnelles acquises dans les démocraties occidentales n'a qu'une portée limitée.

La construction de l'Europe s'accompagne de mise en place de réseaux de so-

2. P.J. Spiro, Embracing dual nationality: International Migration Policy Program, Carnegie Endow ment for International Peace, Occasional Paper 1. 
lidarités transnationales qui défient cette identification à un État-nation. Mais si ces réseaux, même s'ils cherchent à contourner les politiques nationales, agissent en réalité sur les États par l'extérieur, car c'est toujours avec les États que se négocient en dernier ressort les limites de la reconnaissance des différences et, par conséquent, les identités dont l'expression peut être admise comme légitime ${ }^{1}$.

Ces négociations portent sur l'élaboration de nouveaux codes de coexistence en redéfinissant certaines valeurs ou en en renforçant d'autres, afin de combiner ainsi l'" idée "d'État-nation unitaire avec le pluralisme de "fait " des sociétés modernes et sa représentation institutionnelle, d'assurer une continuité historique et de reconnaître les particularités qui surgissent dans l'espace public, en bref de rétablir le lien entre l'État et la société civile pour aboutir à une recomposition du pacte social et politique. Il est question d'un équilibre entre la société civile et l'État, ou d'un lien entre la diversité culturelle et la citoyenneté qui ne porte atteinte ni aux principes civiques, $\mathrm{ni}$ à l'identité finale de la collectivité dans son ensemble.

Focaliser le débat sur la double nationalité ne pouvait que continuer à entretenir l'idée d'un ailleurs qu'on introduit dans la communauté politique et renforcer l'idée d'une minorité nationale avec une identité séparée, qui conserve un statut d'étranger malgré son accession à la citoyenneté légale $^{2}$. Ce qui aboutit à une distinction entre le vrai et le faux citoyen. Alors que la double nationalité pouvait être conçue comme un moyen provisoire et pragmatique d'inclusion dans la communauté politique avec une rhétorique fondée sur-

1. R. Kastoryano, " Participation transnationale et citoyenneté : les immigrés en Europe *, Culture et Conflits, 28, hiver 1997, p. 59-67.

2. Il faut cependant noter que les Aussiedler malgré leur naturalisation à peine arrivés sur le territoire allemand, maintiennent la nationalité du pays de départ (Pologne, Roumanie, ou encore des Républiques d'Asie centrale) Cf. R. Münz, R. Ulrich, " Germany and its immigrants... * art. cité. tout sur la citoyenneté, en faire la pierre de touche du débat sur le changement de loi sur la citoyenneté, malgré l'égalité de droit assurée par la loi, revient à séparer des " nationalités ", à rendre le statut d'étranger permanent et structurel et à exclure l'étranger de l'assimilation.

Le droit à la nationalité des enfants d'étrangers nés eux-mêmes en Allemagne constitue de toute évidence une étape importante dans l'acceptation de l'autre à condition que les structures mentales accompagnent les changements institutionnels et cela grâce au discours social, politique ou médiatique qui conduirait les populations issues de l'immigration, les nouveaux citoyens, à s'identifier avec l'État et ses institutions. Il est difficile de concevoir un État démocratique réellement dépourvu de toute dimension identificatrice ${ }^{3}$.

L'Allemagne, peut-être plus que les autres nations, se trouve exposée aux regards de l'opinion du fait de son passé qui n'a pas encore cicatrisé ses blessures. Les lois sur la nationalité ne devraient pas aller à contre-courant de tout le chemin parcouru par l'Allemagne dans le domaine de la démocratie depuis la fin de la guerre et retarder son processus de "réconciliation " avec les autres États démocratiques. Le vrai défi pour l'Allemagne d'aujourd'hui est donc d'encourager une identification de ses " nouveaux citoyens " avec les institutions allemandes, ses principes fondamentaux et sa Constitution, de les aider à développer un sentiment de responsabilité visà-vis de la vie politique et de leur nouvelle " communauté de destin", fondé sur une nouvelle unité politique et de s'affirmer enfin comme un État-nation en Europe.

3. Cf. Ch. Taylor, - Les institutions dans la vie nationale *; dans Rapprocher les solitudes. Écrits sur le fédéralisme et le nationalisme au Canada (textes réunis par Guy Laforest), Sainte-Foy, Presses de l'Université de Laval, 1992, p. 135153. 
Riva Kastoryano est chercheuse au Centre d'études et de recherches internationales (CERI) de la Fondation nationale des sciences politiques. Elle a publié La France, l'Allemagne et leurs immigrés.
Négocier l'identité (Armand Colin, 1997) et dirigé la publication de Quelle identité pour l'Europe? La multiculturalisme à l'épreuve (Presses de Sciences Po, 1998). 